\title{
Electronic structure of $\mathrm{Ge}$ in $\mathrm{SiO}_{2}$
}

\author{
J P Hagon†, M Jaros† and A M Stoneham \\ Theoretical Physics Division, AERE Harwell, Oxfordshire OX11 ORA, UK
}

Received 29 January 1985

\begin{abstract}
It is argued that one-electron theory is insufficient to account for the origin of the observed spectra of $\mathrm{Ge}$ in $\mathrm{SiO}_{2}(\alpha$-quartz) crystals. A simple model is employed to show that impurity states responsible for ESR spectra of $\mathrm{SiO}_{2}: \mathrm{Gẽ} \mathrm{are} \mathrm{stabilised} \mathrm{by} \mathrm{many-electron}$ polarisation effects associated with the $\mathrm{Ge}$ atom itself and its immediate oxygen neighbours.
\end{abstract}

\section{Introduction}

Previous studies of defects in $\mathrm{SiO}_{2}$ have concentrated on native defects, namely oxygen vacancies and related centres. These studies, as well as the more scarce investigations concerning chemical impurities (Stoneham 1975, Ekenberg et al 1984 and references therein) almost without exception relied on one-electron models. In this paper we consider one of the simplest and best characterised defects in $\alpha$-quartz, a substitutional isoelectronic germanium impurity. We argue that one- electron theory is insufficient to account for the observed spin-resonance spectra of this impurity. We present a brief description of the problems which we have encountered in the course of our efforts to employ one-electron theories, and which we have not been able to overcome. We propose that the observed spectra can be explained if an additional many-electron (correlation) term is included in the Hamiltonian. We employ a simple model which enables us to estimate this correlation effect in terms of interactions between the unpaired electron and the valence electrons localised at the nearest-neighbour oxygen atoms, as well as the impurity germanium atom itself.

\section{2. $\mathrm{SiO}_{2}$ band structure}

If we wish to identify localised impurity levels, we must position them as accurately as possible with respect to the band edges of the host material with due allowance for any polarisation and distortion. It is these transitions between the localised and continuum states that are exploited in material characterisation. One important class of methods, based on the one-electron approximation, makes a particularly close link with the host band structure. What we shall show is that problems with the $\mathrm{SiO}_{2}$ band structure rule out such approaches at the present time.

† Department of Theoretical Physics, The University, Newcastle upon Tyne, UK. 
The methods under discussion separate the one-electron Hamiltonian into the unperturbed part representing the host crystal, $H_{0}$, and a potential $V$ which accounts for the difference between the self-consistent atomic potential of the impurity and host atoms, i.e. $H=H_{0}+V$. The usefulness of this approach has been demonstrated in a number of pseudopotential studies of defects in semiconductors (e.g. Jaros 1982). Analogous studies could be carried out for defects in crystalline $\mathrm{SiO}_{2}$ provided that the eigenfunctions and eigenvalues of $H_{0}$ (i.e. the band structure) can be obtained with sufficient accuracy. Unfortunately the band structure of $\alpha$-quartz has not been characterised adequately, unlike covalent semiconductors. Even the origin of the most prominent features in the optical spectra are still poorly understood, and this leads to problems which we shall discuss.

The most accomplished quantitative study of the band structure of $\alpha$-quartz was presented by Chelikowsky and Schlüter (1977). They employed a non-local oxygen pseudopotential with angular momentum components $l=0$ and $l=1$ fitted to reproduce the atomic valence $2 \mathrm{~s}$ and $2 \mathrm{p}$ electron energies in such a way that the corresponding pseudofunctions of the $\mathrm{O}^{6+}$ ion smoothly matched the real functions at a core radius of $R_{0}=1.4 \mathrm{au}$. Hartree-Fock-Slater local exchange and correlation theory was used to compute the self-consistent potentials which yielded a band gap of $9.2 \mathrm{eV}$. This is in good agreement with the observed threshold of optical transitions at about $10 \mathrm{eV}$. However, the joint density of states at threshold predicted in their calculations was in qualitative disagreement with experiment, i.e. much too low to account for the large and sharp peak in the spectra. Furthermore, the states at the bottom of the conduction band are almost entirely of silicon origin and the fact that the same spectra have been seen in polycrystalline and amorphous $\mathrm{SiO}_{2}$ is difficult to explain (see Mott 1977, Robertson 1983 and references therein).

The predicted dominance of Si-related states at the bottom of the $\mathrm{SiO}_{2}$ conduction band is not peculiar to the pseudopotential method. Since oxygen is highly electronegative, it leaves silicon with a net positive charge. In the framework of one-electron theory, the negatively charged oxygen atom cannot make a significant contribution to the conduction band states since at that range of energies its self-consistent potential is repulsive to electrons. This effect is strengthened by the choice of a pseudopotential in Chelikowsky and Schlüter's calculation constrained to reproduce the valence (atomic) states of oxygen.

It follows that the existing band structures may not be suitable for studies of localised impurity states, split off the lowest conduction band of $\alpha$-quartz. We have, therefore, set up a pseudopotential calculation based on the self-consistent pseudopotential theory, in which we have varied the choice of potential between soft-core (weak and smooth) (Animalu and Heine 1965) and hard core (strong and rapidly varying) (Bachelet et al 1982) potentials, in an effort to understand the mechanism controlling the formation of the conduction band states. However, both these potentials fail to account for the main features of the band structure. In particular the fundamental band gap is reduced to a few $\mathrm{eV}$ in both cases. This means that neither the free-electron-like approach with a truncated weak pseudopotential familiar from the studies on covalent semiconductors, nor the more rigorous local density theory invoking hard-core norm-conserving pseudopotentials, can supply a band structure of $\mathrm{SiO}_{2}$ suitable for detailed impurity calculations based on the perturbative Hamiltonian mentioned above. This implies that many-electron effects may play an important role in determining the conduction band structure of $\mathrm{SiO}_{2}$. Such effects may also allow for much greater participation of oxygen orbitals in the formation of the conduction band in accord with the ideas of Mott (1977). 
The atomic origin of such contributions is likely to be related to strong correlation effects at oxygen. For example in contrast to predictions of one-electron models, many-electron configuration-interaction theory predicts the existence of several excited states of $\mathrm{O}^{-}$ (Massey 1976).

\section{Implications for defect studies: Binding to the isovalent Ge centre in quartz}

In view of our remarks concerning the uncertainties in the band structure of $\mathrm{SiO}_{2}$, a detailed quantitative study of localised states of conduction band character would appear to be outside the reach of present day theory. This is true both for the isovalent impurities like $\mathrm{Ge}$, which are central to the present paper, and for some of the substitutional donors discussed by Ekenberget al (1984). The problems go beyond those of the well understood limitations of effective mass theory because of the relative compactness (radii $\sim 5 \mathrm{au}$ ) of the relatively deep donor states $\left(E_{\mathrm{i}}-E_{\mathrm{c}} \geqslant 1 \mathrm{eV}\right)$ and arise from uncertainties in the nature of the conduction band itself.

For the isovalent impurity $\mathrm{Ge}$ however, the detailed knowledge of the spin-resonance spectra of $\mathrm{Ge}$ in $\mathrm{SiO}_{2}$ can be exploited to provide fresh insight into the origin of the impurity state of $\mathrm{Ge}$. In one-electron theory, based on the perturbative approach, the impurity potential $V$ can be estimated by subtracting self-consistent potentials of $\mathrm{Ge}$ and $\mathrm{Si}$ in the $\mathrm{SiO}_{2}$ lattice. The well resolved spin resonance spectra of Isoya et al (1978) show that there is no significant lowering of symmetry by lattice distortion. The strength of the impurity potential $V$ is dominated by the difference between the ionic (bare) potentials of $\mathrm{Ge}^{++}$and $\mathrm{Si}^{++}$and that of the valence electron's self-consistent charge rearrangement in response to such a perturbation. In particular the ground state is likely to be dominated by the S-component of $V_{\text {bare }}$. However, the difference between the bare potentials is of order $0.01 \mathrm{Ryd}$, i.e. too small to give rise to any localised states, irrespective of the details concerning the changes in the valence charge density and the electronic structure of the conduction band. This is because a short-range core potential forces the wavefunction to vary rapidly at the core radius. Consequently, the kinetic energy is much larger than the one corresponding to hydrogenic states and a high threshold value of $V$ is required to ensure binding.

The origin of the binding can be understood from our studies of isoelectronic impurities in semiconductors, e.g. $\mathrm{N}$ in $\mathrm{GaP}$ and $\mathrm{GaP}_{1-x} \mathrm{As}_{x}$ alloys (Jaros 1984 and references therein). Although the nitrogen one-electron impurity potential is at least an order of magnitude larger than that of $\mathrm{Ge}_{\text {in }} \mathrm{SiO}_{2}$, bound states do not materialise. In the case of $\mathrm{N}$ in $\mathrm{GaP}_{1-x} \mathrm{As}_{x}$, the impurity potential is strong enough to lower the valence selectrons at $\mathrm{N}$ deep below the valence band. This weakens the $\mathrm{sp}^{3}$ covalent bond. As a result there are well-localised resonances in the valence and conduction bands. The valence electrons residing in the semi-localised states near nitrogen can then make virtual transitions to the higher-lying localised states. The gain in the total energy can be expressed in terms of an effective correlation potential. This potential has a maximum at about the nearest-neighbour distance from $\mathbf{N}$ and decays at large distances as $-r^{-4}$. It corresponds to the monopole-induced dipole interaction first discussed by Heisenberg and Bohr (1924); its asymptotic value is more precisely $-\Delta \alpha / r^{4}$ in Rydberg units, where $\Delta \alpha$ is the effective polarisability of the defect atom in units Bohrs ${ }^{3}$. Owing to the relatively long-range character of this potential, extended bound states are more likely to occur. Detailed calculations showed that the combined effect of the short-range oneelectron potential and that of the medium-range correlation potential, is sufficiently 


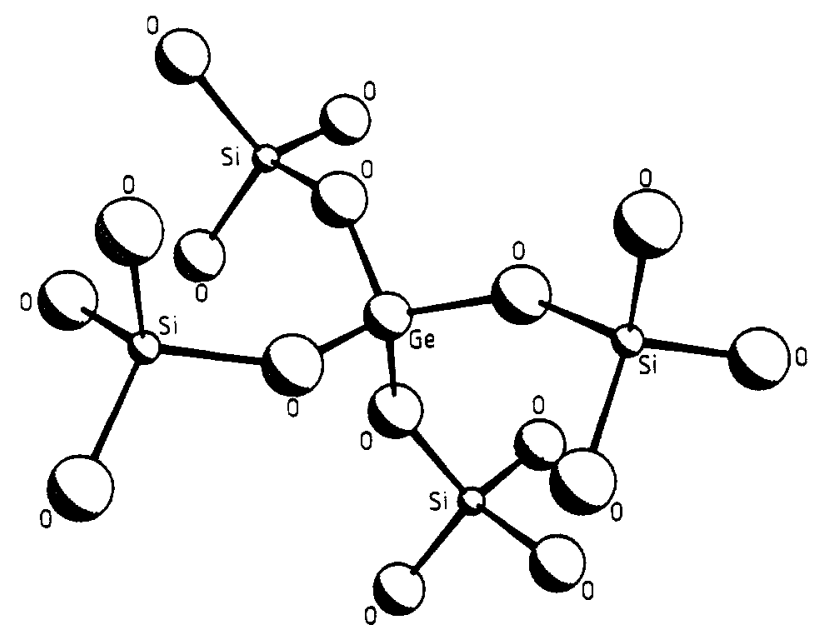

Figure 1. Local geometry of the Ge centre in quartz. This assumes no lattice relaxation. The $\mathrm{GeO}_{4}$ unit is shown together with its four nearest $\mathrm{SiO}_{4}$ units. The viewing direction is $\langle 001\rangle$.

strong to give rise to a bound state of $\mathrm{N}^{-}$. We now apply some of these ideas to the isovalent Ge impurity in quartz ( figure 1) (and consider only the immediate environment, i.e. $\mathrm{GeO}_{4}$ unit). As pointed out by Ekenberg et al, even a weak short-range potential is capable of forming a wave packet in the vicinity of $\mathrm{Ge}$ in $\mathrm{SiO}_{2}$. The localisation of this state could further increase because of correlation effects involving the extra electron and the Ge atom. These effects are due to significant differences in the effective polarisabilities of the $\mathrm{Ge}$ atom and the $\mathrm{Si}$ atom it replaces. We may estimate this difference by use of the Lorentz-Lorenz formula (e.g. Mott and Gurney, equation (13) of chapter 1)

$$
\frac{\varepsilon_{\infty}-1}{\varepsilon_{\infty}+2}=\frac{4 \pi N \alpha}{3}
$$

where $\alpha$ is the polarisability of a molecular unit $(\mathrm{Ge} / \mathrm{Si}) \mathrm{O}_{2}$ and $N$ is the number of molecules per unit volume. We apply the formula to $\alpha$-quartz $\left(\mathrm{SiO}_{2}\right)$ and its $\mathrm{Ge}$ counterpart, germania quartz. The result is $\Delta \alpha=\alpha\left(\mathrm{GeO}_{2}\right)-\alpha\left(\mathrm{SiO}_{2}\right)=6.035 \mathrm{Bohrs}^{3}$. The difference is due to the larger polarisability of the Ge atom. The (slowly moving semilocalised) wave packet mentioned above will therefore see a net correlation potential which at large distances is given by $-\Delta \alpha / r^{4}$ (in Rydberg units). At shorter distances the potential will have a peak at about 2.5 Bohrs (the radius of $\mathrm{Ge}$ atom) and then tend to zero. Our qualitative calculations suggest that the strength of this potential, although small, will exceed the weak one-electron impurity potential of Ekenberg et al who reported that a potential slightly stronger than theirs would give rise to a bound state. However, it is clear that this $r^{-4}$ potential is not sufficient to cause binding itself. Use of standard results (Stoneham 1975 para. 9.2) show that an unscreened $r^{-4}$ potential, modified to an $\left(r^{4}+b^{4}\right)^{-1}$ form so as to saturate with $b \leqslant 1.54$ Bohrs could indeed bind an electron. Inclusion of screening (note that $\varepsilon_{\infty}^{-2} \simeq 0.17$ ) means that additional binding is needed.

Some of the extra binding may come from symmetric lattice distortion, though no lowering of symmetry is noted. A much greater degree of localisation would result if interactions with the nearest-neighbour oxygen atoms were included, for the weakly- 
bound electron will be moving slowly enough such that it distorts the largely atomic-like non-bonding $\mathrm{p}$-electrons on each oxygen atom. This also results in a correlation potential which is in fact even stronger than that due to germanium (figure 2). The potential was calculated by assuming that the (one-electron) Schrödinger equation for each oxygen valence electron is modified by a (dipole) term of the form $-2 r \cos \theta / R^{2}$, where $R$ is the radial distance of the extra electron and $r, \varphi$ are the co-ordinates of the oxygen valence electron. This perturbation was treated according to the procedure of Sternheimer (1954) and first-order corrections to the Hartree-Fock orbitals were then found. These corrected electron orbitals were then used to obtain the desired potential following, for example, the method of Callaway (1957). The oxygen atoms may also play a crucial role in determining the ground state wavefunction, which is known from spin resonance spectra to be localised off-centre in a germanium sp orbital. Furthermore, the direction of the sp hybrid is observed to coincide with the bisector of one of the $\mathrm{O}-\mathrm{Ge}-\mathrm{O}$ angles. Spin resonance studies also indicate that the $\mathrm{GeO}_{4}$ unit is similar to the $\mathrm{SiO}_{4}$ unit both before and after the trapping of an electron (i.e. there is very little lattice distortion). That the ground state wavefunction has a component along an $\mathrm{O}-\mathrm{Ge}-\mathrm{O}$ bisector can be explained using the present theory. The $\mathrm{Ge}-\mathrm{O}$ bonds are much less polarisable than the non-bonding oxygen $p_{\perp}$ electrons, and so the oxygen correlation potential is stronger along a line perpendicular to the bond. Using a radial potential of similar form to figure 2 one may perform an approximate calculation with a point charge situated in the vicinity of the $\mathrm{GeO}_{4}$ unit, and vary the ratio of bond axial polarisability to perpendicular polarisability. It turns out that if this ratio is anywhere in the range 0.4 to 0.7 and the point charge is in a range $0.3 a-0.6 a$ ( $a=\mathrm{Ge}-\mathrm{O}$ bond length) then the most favoured direction is along an angle bisector. For a similar range for the polarisability ratio but with the radial distance of the point charge from the Ge atom increased up to $1.1 a$ then the $\langle 110\rangle$ direction is most favoured with the angle bisector $\langle 100\rangle$ being second best. However the $\langle 110\rangle$ direction is unstable with respect to electron repulsion so it is likely that here the $\langle 100\rangle$ angle bisector direction is again the most stable. The peak in the radial probability density of the unpaired electron's sp hybrid occurs at about $0.8 a$ $\left(=2.4 a_{0}\right)$ which is consistent with the above theory. Also as the $\mathrm{GeO}_{4}$ unit is not exactly tetrahedral there will be different values of the $\mathrm{O}-\mathrm{Ge}-\mathrm{O}$ bond angle in each

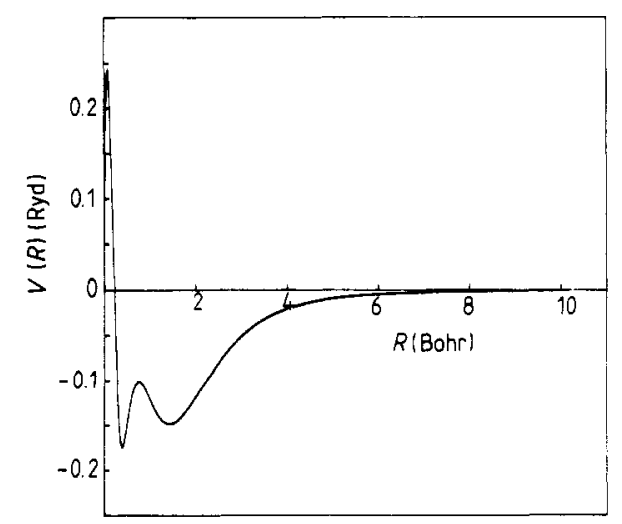

Figure 2. Oxygen correlation potential. At large distances this behaves as $-1 / r^{4}$. The complex structure of this potential for $R<2$ au is dependent on the internal electronic structure of the oxygen atom. 
$\mathrm{GeO}_{4}$ unit. Due to short-range electron repulsion, an electron localised to some extent in the most open angle will correspond to the most stable centre. The spin resonance studies of Isoya et al confirm this. They observe two distinct centres $\mathrm{Ge}$ (I) and $\mathrm{Ge}$ (II). The energy difference between these states is small enough for both to be populated, but large enough for a temperature dependence to be observable between $\sim 15 \mathrm{~K}$ and room temperature. Other unobserved states corresponding to the other less open angles may also exist. Our model also agrees with the observation of Isoya et al that a substantial portion $(\sim 50 \%)$ of the unpaired electron density is not localised at the Ge atom since the correlation potential at the oxygen ligands is substantial.

\section{Conclusions}

We have drawn attention to the difficulties concerning the implementation of oneelectron theory in any detailed quantitative study of impurities in $\alpha$-quartz. In particular, we find that the most accomplished pseudopotential band structure calculations are not accurate enough to describe the key features (e.g. the fundamental gap) of $\mathrm{SiO}_{2}$. We have considered an isoelectronic germanium impurity in $\mathrm{SiO}_{2}$ and suggested that the simple many-electron model recently developed for isoelectronic impurities in covalent semiconductors can be used to explain in qualitative terms the properties of this defect revealed by spin resonance studies. The ground state of the unpaired Ge electron is stabilised by correlation effects between the extra electron, the Ge atom and the proximity of the observed ground state. Our study indicates that many-electron effects may play an important role in determining the conduction band states in perfect and imperfect $\mathrm{SiO}_{2}$.

\section{References}

Animalu A O E and Heine V 1965 Phil. Mag. 121249

Bachelet G B, Greenside H S, Baraff G A and Schlüter M 1981 Phys. Rev. B 244745

Callaway J 1957 Phys. Rev. 106868

Chelikowsky J R and Schlüter M 1977 Phys. Rev. B 154020

Ekenberg U A, Robertson J and Dow J D 1984 Phys. Rev. B 292216

Heisenberg W and Bohr M 1924 Z. Phys. 23388

Isoya J, Weil J A and Claridge R F C 1978 J. Chem. Phys. 694876

Jaros M 1982 Deep Levels in Semiconductors (London: Adam Hilger)

1984 Solid State Commun. 51411

Massey H S W 1976 Negative Ions (Cambridge: CUP)

Mott N F 1977 Adv. Phys. 26363

Mott N F and Gurney R W 1948 Electronic Processes in Ionic Crystals (Oxford: Clarendon)

Robertson J 1983 Adv. Phys. 32361

Sternheimer R M 1954 Phys. Rev. 96951

Stoneham A M 1975 Theory of Defects in Solids (Oxford: Clarendon) 